\title{
LA IMPORTANCIA DE LA RELACIONES TEMPRANAS Y EL APEGO EN LOS NIÑOS CON NECESIDADES ESPECIALES
}

\section{The Relevance of Early Relationships and Attachment in Children with Special Needs}

\begin{abstract}
Andrea Mira O. ${ }^{\mathrm{i}}$
RESUMEN

El establecimiento de un apego seguro es un factor que promueve el desarrollo y bienestar del niño y su familia. Es por esto que en este trabajo hemos querido ligar el concepto del apego con los niños con necesidades especiales, y el cómo las relaciones tempranas pueden potenciar su desarrollo motor, cognitivo y socio-emocional. Del mismo modo queremos plantear la necesidad que existe de incluir el apego como eje central de las intervenciones tempranas, lo cual puede realizarse a través del empoderamiento de los padres, entregándoles claves que les permitirán tener interacciones más cálidas y significativas, reconociendo el estilo y necesidades de su hijo, logrando potenciar su sensibilidad y la capacidad para regularlos y establecer interacciones que van a potenciar su desarrollo y aprendizajes.
\end{abstract}

Palabras Claves: Apego seguro, niños con necesidades especiales, regulación.

\begin{abstract}
The establishment of a secure attachment enhances the family and child development and wellbeing. This is why in this essay we wanted to bind the attachment theory with the children with special needs and show how the early relationships can promote the motor, cognitive and socio-emotional development. In the same way we wanted to stress the need of including the attachment as a core aspect in the early interventions, through the parent's empowerment by giving them the cues that would let them have warmer and more significant interactions, being able to recognize their child style and needs, and as a result of this they can achieve a better sensibility and the ability to regulate them and establish interactions that can favor their development and knowledge.
\end{abstract}

Key Words: Secure attachment, children with special needs, regulation.

\footnotetext{
i Terapeuta Ocupacional, Licenciada en Ciencias de la Ocupación Humana. Universidad de Chile. Diplomado en Neuropsicología, Universidad Católica, Magister en Salud Mental Infantil @), Universidad del Desarrollo.

Formación en Integración Sensorial. Cargo Terapeuta Ocupacional en centro Althea y Amancay y en la unidad de psiquiatría infantil Clínica las Condes. Email: andreamira@yahoo.com
} 


\section{INTRODUCCIÓN}

¿Qué pasa cuando la imagen del bebé idealizado no se cumple, específicamente cuando los padres reciben la noticia de que su hijo presenta alguna dificultad o un retraso en su desarrollo psicomotor? Esta noticia puede ser devastadora e inquietante, apareciendo sentimientos de temor, confusión, tristeza e incluso puede producirse un bloqueo emocional y una sensación de no saber que hacer. Los padres o cuidadores primarios se sumergen en un mundo donde abundan las terminologías médicas, las cuales pueden ser difíciles de comprender, más aún, deben tomar decisiones en relación a los cuidados e intervenciones que debe recibir su hijo. Por otro lado, tenemos a este niño que, como cualquier otro, requiere del amor, apoyo y contención de sus padres, y que independiente de su diagnóstico es un ser con características y necesidades propias. Todos estos factores hacen que sea interesante analizar el tema de las relaciones tempranas y apego entorno a los niños con necesidades especiales y sus cuidadores primarios.

Al hablar de niños con necesidades especiales nos referimos a cualquier niño que necesite ayuda adicional debido a un problema médico, emocional o en sus aprendizajes. Estos niños pueden requerir del uso de fármacos, terapia u otra asistencia para potenciar su desarrollo, apoyos que otros no suelen necesitar o solo los requieren de forma esporádica. En el ámbito de las necesidades especiales encontramos un amplio espectro de condiciones, donde los apoyos o intervenciones necesarias pueden ser mínimos o necesarios para el sustento vital del menor. Dentro de este grupo se encuentran niños con trastornos generalizados del desarrollo, prematuridad, parálisis cerebral, síndrome de Down y otros síndromes genéticos, enfermedades metabólicas, problemas de regulación, alergias alimentarias, retraso del desarrollo psicomotor, síndrome fetal alcohólico, entre otros.

La crianza de un niño es un proceso de aprendizaje continuo, no exento de dificultades y confusiones, problemáticas que se acrecientan en el caso de las familias de niños con necesidades especiales, ya sea por las características temperamentales del bebé, por su fragilidad y condiciones de salud y/o por las capacidades de adaptación de la familia. Dentro de las experiencias que hemos observado en la clínica se evidencian ciertas dinámicas en las relaciones tempranas entre padres y bebés con necesidades especiales y como estas dinámicas van influyendo en el desarrollo del niño y su interacción con el mundo. El estrés y temor que rodean a estos padres suele desencadenar patrones intrusivos y controladores para interactuar con sus hijos, lo cual coincide con datos encontrados en la literatura donde se señala, por ejemplo, que las madres de niños prematuros suelen ser menos sensibles y más controladoras, lo que se intensifica cuando ellas han sido expuestas a grandes cantidades de estrés en el periodo perinatal $^{1}$. En madres de niños pequeños para la edad gestacional, también se presentan patrones intrusivos, donde predominan las descoordinaciones y un involucramiento negativo con el hijo ${ }^{2}$.

Para comprender las dinámicas que se presentan en las familias de niños con necesidades especiales es importante conocer acerca de las interacciones entre padres y niños que presentan una trayectoria de desarrollo dentro de la normalidad. En el área de las relaciones tempranas se ha estudiado con gran interés el apego y su efecto en el desarrollo y salud mental de los niños. La Teoría del Apego comienza a esbozarse alrededor de los años cuarenta de la mano de su creador John Bowlby, no obstante, no es hasta los años setenta que sus planteamientos empiezan a tener una importante influencia en el área de la salud mental. Podemos definir apego como una propensión innata de los seres humanos a establecer relaciones afectivas intensas y duraderas con una figura cercana, donde ésta le entrega seguridad y protección, especialmente en 
situaciones de estrés y vulnerabilidad, considerando estrés como cualquier estado que desequilibre la homeostasis biológica, afectiva y social del organismo. Por lo tanto, el apego es el lazo afectivo que se establece entre el niño y una figura específica, que une a ambos en el espacio, perdura en el tiempo, se expresa en la tendencia estable a mantener la proximidad y cuya vertiente subjetiva es la sensación de seguridad ${ }^{3}$.

La Teoría del Apego permite vislumbrar la relación entre el recién nacido y sus padres como una relación bidireccional, donde el bebé contribuye desde los primeros momentos a esta interacción. Además se sabe que los recién nacidos poseen un importante número de complejas habilidades psicológicas y sociales, y que los cuidadores y sus bebés desarrollan, desde los primeros minutos de vida del niño, un patrón dinámico de coordinación y sintonía, una regulación afectiva y fisiológica mutua, y establecen una comunicación socio-afectiva ${ }^{4}$.

Las interacciones afectivas entre la madre y el bebé influyen en el desarrollo de los sistemas de regulación cerebral, responsables a su vez de la regulación de la conducta, cognición y afectos del bebé 5 . A través de los encuentros afectivos entre la madre y el infante, se empieza a desarrollar en éste un conjunto de habilidades y mecanismos para enfrentar y regular el estrés, las emociones, las situaciones novedosas, el aprendizaje y los estados mentales, en épocas posteriores (schORE, 2001). Los bebés no poseen todas las habilidades para regularse, es por esto que los cuidadores actúan como reguladores externos. Esto implica que entre ambos se va creando una vía de comunicación, donde el bebé va expresando sus necesidades a través de diversas conductas (por ejemplo, llanto, vocalizaciones, entre otras) que buscan provocar una respuesta en el adulto. A su vez los padres deberán tener la capacidad de percibir, interpretar y responder contingentemente a estas señales (lo que se conoce como “sensibilidad materna') y, en la medida que los cuidadores van ayudando a su hijo a bajar los niveles de estrés, éste podrá explorar y aprender a través de sus experiencias.

Las conexiones entre los padres y su bebé van ocurriendo a través de las interacciones cotidianas - en la alimentación, en el juego o cuando le entregan cuidados a sus hijos - en donde los padres van aportando un soporte y apoyo, calmando o activando al bebé según vaya siendo necesario. Es así como en los primeros meses se va fortaleciendo un patrón de regulación mutua, tanto de los estados fisiológicos como afectivos. Esta sintonía y sincronía entre padres y bebé no implica que vivan en un estado continuo de coordinación y armonía perfecta, sino que lo fundamental está en la capacidad del cuidador de reparar la descoordinación que se ha producido, para así retomar el flujo armónico de interacción. De este modo los niños podrán predecir con mayor facilidad la respuesta de sus padres ante las situaciones de estrés y los buscarán a ellos como fuente de confort y seguridad.

El desarrollo y maduración cerebral están determinados por factores genéticos y por la experiencia, por lo tanto, el ambiente físico y social en el que esté inmerso el niño irá moldeando estos procesos, y es por esto que las interacciones tempranas y el estilo de apego que se forme con sus cuidadores influirán directamente en el niño. En esto se conjugarán factores tales como las características propias del bebé, la capacidad de los padres para ajustarse al estilo de su hijo, las respuestas de éstos frente al estrés y la calidez y calidad de las interacciones, que irán afectando el desarrollo cerebral de sus hijos. Y si en las interacciones cuidador - niño predominan las coordinaciones y un buen ajuste entre ambas partes, el proceso tendrá como resultado el desarrollo de un apego seguro, donde tendremos un niño que buscará a sus padres en 
momentos de estrés para obtener confort y apoyo y se ira generando un modelo mental de confianza que va a potenciar su seguridad y desarrollo.

Viendo el impacto que tiene el establecimiento de un apego seguro entre los padres y sus hijos, se hace necesario analizar la relación entre padres y niños con necesidades especiales en sus primeros años de vida, más específicamente, mostrar cómo podemos hacer que las relaciones tempranas con las figuras de apego promuevan la regulación, aprendizaje y desarrollo.

En este caso las relaciones tempranas estarán inmersas en un contexto complejo. Los padres se enfrentarán a sentimientos y situaciones contradictorias, donde la estimulación o las intervenciones terapéuticas van a ir tomando un eje central en la dinámica familiar. Sumado a esto, se les presentarán dificultades en torno al cuidado y crianza de sus hijos, por sus características temperamentales o de la patología de base. Todos estos factores van a ir tiñendo la percepción que tienen los padres de su hijo y estas percepciones influyen en la forma en que el padre se relaciona con su bebé. Estudios han mostrado que las madres tratan a sus hijos de acuerdo a las percepciones que tengan de éstos y sus expectativas y, que las creencias en relación al diagnóstico del bebé van determinando las percepciones y conductas hacia su hijo, independiente de las reales características y conductas de estos ${ }^{6}$. Es por esto que es de gran importancia que los padres vayan conociendo las potencialidades, desafíos y formas de reaccionar e interactuar.

Si logramos fortalecer el apego y las interacciones tempranas entre los niños con necesidades especiales y sus padres, ya estaríamos potenciando el desarrollo físico, cognitivo y social del menor, aspectos que suelen estar dentro de los objetivos de las intervenciones tempranas de diversos profesionales, como terapeutas ocupacionales, kinesiólogos, psicólogos y fonoaudiólogos. A continuación profundizaremos en estos aspectos y en el cómo podemos involucrar y potenciar un rol activo de los padres en las intervenciones tempranas, para así obtener resultados positivos y consistentes entorno al desarrollo y bienestar del niño y su familia.

\section{METODO}

Como mencionamos anteriormente, dentro del grupo de niños con necesidades especiales, tenemos una amplia gama de diagnósticos y problemáticas, donde el factor común es que ellos necesitan de un apoyo extra, ya sea el uso de fármacos o intervenciones terapéuticas para potenciar su desarrollo y bienestar. En general, los diagnósticos resumen los signos y síntomas que manifiestan, no obstante, no nos entregan información de los procesos que están relacionados con las dificultades y desafíos que presentan, más específicamente como los niños reciben, procesan y responden a la información del medio ${ }^{7}$. Estos tres aspectos son el centro de las habilidades del niño para pensar, sentir e interactuar (GREENSPAN, 1998), y van formando su estilo para relacionarse con el medio en sus primeros años de vida. Esto quiere decir que podemos tener dos niños con el mismo diagnóstico, pero con conductas, necesidades y potencialidades muy distintas.

En los infantes que presentan dificultades en su desarrollo, estos tres aspectos (el como recibe, procesa y responde a los estímulos) toman especial importancia dado que le estarán dando forma a sus interacciones con el medio físico y social, influyendo en las relaciones tempranas con sus padres. La clave para formar un apego seguro, está en la capacidad de los padres para leer las 
señales y necesidades de sus hijos y responder a tiempo y adecuadamente, pero en este caso, ellos tendrán que ver más allá del diagnóstico para conocer cómo su hijo toma la información del medio, cómo la procesa y logra responder a ésta, de este modo irán tomando significado sus conductas y les será más fácil responder ante sus necesidades.

Es en este punto donde debemos detenernos y ver cómo estos aspectos pueden incluirse dentro de los programas de intervención temprana, donde los profesionales de la salud deberíamos favorecer el desarrollo integral del niño a través del involucramiento y empoderamiento de los padres. Esto por medio de la entrega de herramientas para que ellos al interactuar y jugar con sus hijos, logren aumentar su sentido de eficacia (al saber como cuidarlos, estimularlos e interactuar con ellos), su sensibilidad y puedan encontrarse con ellos en esta interacción. Diversas investigaciones han demostrado que los padres que reciben apoyo y guía en como potenciar sus interacciones con sus bebés, logran tener respuestas más sensibles hacia las necesidades de sus hijos ${ }^{8}$. Además, las intervenciones que promueven interacciones positivas entre la madre y su bebé pueden reducir los riesgos de un pobre desarrollo del niño ${ }^{9}$.

La gran pregunta es ¿Cómo podemos favorecer la sensibilidad de estos padres y el establecimiento de un apego seguro? o bien ¿Cómo podemos favorecer los procesos de regulación en esta diada y así potenciar el desarrollo del bebé? Al unir la teoría con la práctica hemos podido ver varios aspectos que son fundamentales, dentro de los cuales están: enseñarles a los padres a observar a sus hijos, identificar sus ritmos, reconocer los signos de estrés y qué estímulos lo confortan y cuáles lo desregulan, conocer estrategias de manejo ante situaciones conflictivas y fomentar las relaciones con su bebé en el presente estando atento a sus necesidades.

Analicemos estos aspectos paulatinamente, partiendo con el observar y el interactuar en el presente. En los niños con necesidades especiales, es muy común que los padres tengan preocupaciones en cuanto a la evolución y desarrollo de su hijo, por lo que al interactuar con ellos es muy probable que los padres estén pensando si los están estimulando bien o si sus acciones tendrán un efecto positivo en las destrezas de su bebé. Para que los padres logren estar en el presente y observar a su hijo, deben focalizar su atención en lo que están haciendo en el momento y ellos pueden hacer esto sólo a través de la interacción con su hijo que ocurre en el juego o en otras acciones, en donde se favorezca la mutua aceptación total, sin expectativas que podrían llevar la mirada más allá del momento ${ }^{10}$.

Es fundamental que estos encuentros afectivos e interacciones entre el cuidador y el niño sean dentro del presente. Cuando el cuidador presta atención al futuro de su hijo mientras interactúa con él, en realidad éste no se encuentra con su niño en la interacción debido a que su emoción y atención no están en el encuentro sino que están en otro lugar. Esto pondrá una barrera entre ambos y le será muy difícil a los padres percibir y, más aún, comprender las conductas de su bebé. Si los padres están interactuando con su hijo pero pensando sólo en los resultados que estás acciones tendrán o su atención está en otras preocupaciones (por ejemplo, problemas laborales, económicos, entre otros) ellos no lograrán ver a su niño y leer las claves que él les está entregando. Si esto ocurre constantemente, éste se va volviendo "invisible" para los padres, por lo cual, cada vez les será más difícil detectar y responder a tiempo a las señales de su hijo y no les será fácil regularlo. Esto va produciendo descoordinaciones entre los cuidadores y el bebé, que deben ser detectados por el terapeuta para posteriormente ser abordados dentro de las intervenciones. 
Fomentar el goce entre la interacción del niño con sus padres, dejando atrás las expectativas y preocupaciones, debe ser una prioridad dentro de nuestro actuar. Podemos mostrarles a los padres que pueden observar en la interacción cómo su hijo va respondiendo a sus acciones, qué estímulos les gustan más, cuáles son sus tiempos y necesidades. Este observar es un proceso continuo que les permitirá a ellos tener un buen ajuste con las características de su hijo y serán capaces de proveerles de experiencias óptimas acorde a sus capacidades y tolerancia, y así, podrán disfrutar de una relación que fluye con más facilidad. La capacidad de observar unida a una adecuada mentalización de sus hijos, entendiendo mentalizar como al proceso mediante el cual uno interpreta las acciones de los otros y de nosotros mismos, en base a los estados mentales intencionales (por ejemplo, deseos, necesidades, creencias, sentimientos, entre otros), les permitirá a los padres tener un mejor entendimiento de las conductas de su hijo, logrando ver las cosas desde el punto de vista del niño, sin que las señales de éste sean distorsionadas por las necesidades y preocupaciones de los padres. Así los cuidadores irán viendo y tratando de entender porque su hijo realiza ciertas acciones y como se sienten ellos respecto a éstas, por ejemplo, si el niño está irritable y llora, los padres se pueden preguntar el por qué de este llanto, las causas y los motivos del niño para llorar, por otro lado ellos, tendrán que analizar que les produce a ellos esta conducta (inseguridad, temor, insatisfacción o bien tranquilidad porque saben que lo pueden calmar).

Por esto se hace necesario entregar orientación y estrategias a los padres para que puedan identificar las conductas o expresiones de su hijo y así reaccionar a tiempo. Por ejemplo, mostrándoles cómo reconocer los signos de estrés, tales como conductas desorganizadas (llanto excesivo, inquietud motora, entre otras), aumento de la frecuencia cardiaca y respiratoria, cambios en la coloración de la piel y conductas de evitación (mirar hacia otro lado, evitar contacto visual). Éstos nos entregan información muy valiosa, y no sólo en los recién nacidos, sino que también cuando son más grandes, especialmente cuando no han desarrollado un nivel adecuado de lenguaje verbal que permita expresar su malestar. Al utilizar esta información los padres podrían actuar a tiempo para calmar a su hijo, evitando que éste caiga en el llanto incontrolable. También podemos ayudarlos a identificar cuando su hijo está en el momento ideal para la interacción, por ejemplo, cuando éste tiene expresiones faciales placenteras, fija la mirada en la cara de los padres, comienza a vocalizar, se muestra interesado por los objetos y personas e intenta tocarlos y sus movimientos corporales son armónicos.

De la misma manera debemos enseñarles a los padres a reconocer que estímulos favorecen la regulación y cuáles no. Les podemos explicar que cada niño procesa y responde a los estímulos de manera particular y esto va a estar dado por características temperamentales, factores ambientales y en algunos casos por particularidades de su cuadro. Por ejemplo, en el caso de los niños prematuros donde su sistema nervioso central se satura rápidamente con la estimulación y su respuesta frente a los estímulos suele ser impredecible, vamos a tener niños que requerirán de estímulos suaves e idealmente de una modalidad sensorial a la vez. Dentro de las sesiones con los padres debemos mostrarles que estímulos son favorecedores de la regulación, como la contención (al sostenerlos en brazos, envolverlos en una manta), la postura que favorece la flexión, la voz de la madre, el tacto profundo, los movimientos rítmicos y suaves, entre otros, para que ellos vayan integrando estos en su interacción con el infante y a la vez que vayan observando la respuesta de su hijo para poder identificar cuales estrategias son más efectivas. También será importante mostrarles conductas que están relacionadas con la autorregulación del bebé (que están relacionados con los esfuerzos propios del niño para volver a la calma), como 
por ejemplo, la succión del pulgar o chupete, el intentar ir hacia la flexión y en casos más extremos cuando el niño ya no puede lidiar con los estímulos ambientales se mostrará pasivo, somnoliento y/o se quedará dormido. Esta información les permitirá a los padres adaptarse a las características de su hijo, favoreciendo un buen ajuste entre ambos.

Dentro de los niños con necesidades especiales vamos a tener niños que suelen estar en un estado de hiper-alerta, que se sobre-estimulan con facilidad, les es difícil regular sus ciclos de sueño y vigilia, generalmente son irritables y lloran con gran facilidad. Estos niños presentan un gran desafío para los padres y su entorno, especialmente por su difícil manejo y por el alto nivel de estrés que generan en la familia. En la otra cara de la moneda, podemos tener niños con un alerta baja, que parecen somnolientos, son muy pasivos y sus interacciones con los objetos o personas son escasas. En estos casos debemos reforzar el que los padres puedan identificar si sus hijos requieren ser calmados para poder bajar su alerta y nivel de actividad o si necesitan estímulos que los activen y despierten su atención. En el primer caso, un factor crucial para lograr la regulación y vuelta a la calma de estos niños es que los padres estén tranquilos y que actúen tempranamente una vez que han visto los primeros signos de estrés, que lleven a sus hijos a un ambiente bajo en estímulos (con luz tenue, silencioso) y que les den tiempo para volver a la calma. En los niños con un estilo más pasivo, los padres deberán realizar importantes esfuerzos para entregarles estímulos que aumenten su alerta y favorezcan así su interés por interactuar con el medio. Van a necesitar apoyo para enriquecer su exploración y acciones con el medio.

En la medida que los padres vayan identificando el estilo y ritmo de su bebé, van a ir siendo más efectivos al regularlo. El como el niño procesa y responde a los estímulos va a afectar su nivel de alerta, estado emocional, atención y capacidad de actuar, y además está íntimamente ligado con la capacidad del niño para autorregularse ${ }^{11}$. Los niños con necesidades especiales suelen presentar mayores dificultades para autoregularse y mantener estados de alerta óptimos para la interacción, y requieren de más ayuda externa para poder lograrlo, esto debido a posibles compromisos de estructuras del sistema nervioso central (sCHORE, 2001).

Este proceso de regulación es fundamental para que el niño logre tomar interés en el medio, y una vez que esto es posible, podrá focalizar su atención y sus afectos en la relación con sus padres. Si analizamos esto con una mayor detención, vemos que acá hay un factor muy importante para poder potenciar la relación de este niño con sus padres y así favorecer la sintonía y sincronía entre ellos. Esto le va a ir permitiendo al niño complejizar sus interacciones, tanto con sus figuras cercanas como con el medio físico que los rodea, lo que tendrá un impacto positivo en su desarrollo social, cognitivo, emocional y motor. Lo que significa que el niño va a ir desplegando una amplia variedad de destrezas en estas interacciones, lo cual va a favorecer su desarrollo y maduración cerebral. Más allá de estimular destrezas aisladas, por ejemplo, que el niño manipule objetos, o que desarrolle la pinza fina, o obtenga mejores reacciones de equilibrio, debemos potenciar sus aprendizajes favoreciendo que el niño mantenga estados de alerta óptimo, a través de la regulación de sus padres y de sus capacidades de autorregulación. Esto, a su vez, le va a permitir estar atento al medio físico y social para así poder actuar con estos, lo cual va a facilitar el desarrollo de habilidades funcionales.

Para ejemplificar esta situación citaremos dos casos que hemos observado en la práctica clínica. El primero, es el de una niña de dos años y con un cuadro genético que afecta diversas áreas del desarrollo. Ambos padres mostraban una gran preocupación por su hija, pero ésta estaba centrada en el retraso de la adquisición de ciertos hitos del desarrollo psicomotor, especialmente en el área 
del lenguaje y en relación a ciertas destrezas motoras. Al conocer a esta niña, observamos sus dificultades para regular y organizar su conducta, ella se irritaba con facilidad y le era muy difícil expresar sus necesidades, lo cual terminaba, generalmente, en un llanto inconsolable. La intervención, en este caso de la terapeuta ocupacional, con los padres estuvo relacionado con el que empezaran a jugar e interactuar con ella disfrutando el momento, por otro lado, debimos entrar en un proceso de reconocimiento de las señales de su hija para que ellos pudieran actuar rápidamente favoreciendo su regulación. Esta ayuda externa para la regulación que entregaban los padres, fue fomentando el desarrollo de la autorregulación de su hija, siendo capaz de expresar sus necesidades sin caer inmediatamente en el llanto y además, logró aumentar los tiempos de espera para la satisfacción de sus necesidades. Estos padres fueron poco a poco desarrollando una mayor sensibilidad ante las señales de estrés de su hija y aprendieron ciertas técnicas que promueven la regulación, lo cual permitió que ella lograra estar más dispuesta a interactuar con el medio, creando las bases para el desarrollo de habilidades más complejas.

Otro caso que nos puede ayudar a comprender todos los conceptos mencionados anteriormente, es el de unas trillizas prematuras de un mes de edad corregida, donde una de ellas no logró sobrevivir. En este caso nos vimos enfrentados a un contexto complejo donde ambos padres estaban lidiando con la pena y duelo de una pérdida, y con el miedo y aprehensión de que a sus otras dos hijas les fuera a suceder algo. Fuimos observando como las tocaban, si vocalizan y si centran su mirada y atención en ellos al cargarlos, mientras les cambian pañales o los alimentan. Es así como fuimos viendo que estos padres intentaban con todos sus esfuerzos controlar las situaciones que pudieran afectar la fragilidad de sus bebés, por ello les era difícil observar las conductas que sus propias hijas estaban expresando. Para aumentar la confianza en ellos y el sentido de eficacia como padres, fuimos viendo algunas técnicas de manejo que facilitarían los cuidados de estos bebés (pautas de estimulación), por ejemplo, algunas formas para tomarlas que favorecerían su regulación, el reconocer los signos de estrés, el descubrir qué estímulos eran más adecuados para cada una y el poder adaptar sus forma de interactuar con ellas de acuerdo a sus necesidades y estilos y, principalmente el relacionarse en el presente. En este caso es necesario que las pautas de estimulación sean entorno a la calidez de la interacción entre ellos, evitando el realizar ejercicios repetitivos, ya que estos no tendrán mayor significado para los padres ni para los bebés. Esto último produce un aprendizaje superficial y automático para ambas partes, y no tiene el mismo impacto que aquellas que enriquezcan el apego como prioridad.

La clave está en que los padres observen y conozcan a su hijo y que puedan adecuarse a su estilo, que conozcan sus intereses y así puedan entrar y enriquecer su juego e interacción con el mundo. Tenemos entonces que con la regulación externa entregada por los padres, los niños van a ir desarrollando la capacidad de autoregularse y esto va a abrir una ventana hacia los aprendizajes.

\section{DISCUSION}

En este trabajo hemos pretendido realizar un puente entre los conceptos de apego y los niños con necesidades especiales. Para realizar esta unión de conceptos debimos describir las interacciones entre padres y niños sanos y para luego ahondar en el impacto positivo que tiene el establecimiento de un apego seguro en el desarrollo y maduración cerebral del infante y más específicamente el impacto que podrían tener en los niños con necesidades especiales. El 
objetivo del trabajo estaba relacionado con el analizar el como podemos hacer que las relaciones tempranas con las figuras de apego fomenten o promuevan la regulación, desarrollo y aprendizajes de los niños. Dentro del análisis que realizamos hay ciertos puntos que son fundamentales y es importante destacarlos, como el que los padres puedan ver más allá del diagnóstico de su hijo para conocer y reconocer sus estilos, necesidades, potencialidades y cómo ellos van recibiendo, procesando y respondiendo a los estímulos del medio. Así mismo, el potenciar las interacciones en el presente, dejando atrás las expectativas y preocupaciones.

Dada la relevancia de las relaciones tempranas en los infantes y el como un apego seguro puede potenciar el proceso de regulación y desarrollo, es que consideramos que los profesionales de la salud debiéramos tener al apego como eje central de nuestro quehacer en las intervenciones tempranas. Fomentando que los padres tomen un rol activo, para lo cual (padres y profesionales de la salud) debemos compartir nuestros conocimientos y experiencias en relación a aspectos concernientes al cómo observar, conocer, interactuar y estimular a los niños, dentro de un contexto de interacciones cálidas y significativas.

Un tema que sería interesante analizar es el impacto económico que podrían tener los programas de intervención temprana, públicos y privados, que tuvieran el desarrollo de un apego seguro y el empoderamiento de los padres como eje central. Es decir, cuál sería la relación costo-efectividad de las intervenciones tempranas que consideraran el fomento del apego y el darles un rol más activo a los padres, de modo tal que ellos pudieran potenciar el desarrollo y bienestar de su hijo en el día a día. Este empoderamiento de los padres (entregar un rol activo a los padres en relación a la toma de decisiones y acciones concernientes a su hs hijos, aumentando el control sobre las situaciones), lo lograríamos a través del involucramiento activo de ellos en las sesiones, donde los diversos profesionales que trabajamos en esta área (terapeutas ocupacionales, psicólogos, pediatras, kinesiólogos, fonoaudiólogos, entre otros) los podemos guiar y orientar en este proceso de aprendizaje, regulación, estimulación y desarrollo. Esto requiere de un trabajo en equipo, para poder trabajar todos con una mirada similar, incluyendo y acogiendo las necesidades y preocupaciones del niño y su familia. De este modo podríamos combinar la labor de lo profesionales con el de los cuidadores del menor, de manera tal que podríamos aumentar la intensidad de los cuidados destinados a promover el desarrollo y bienestar, y disminuir el costo que significan estas intervenciones para el gobierno o para cada familia.

Quizás así podríamos mejorar el acceso a intervenciones tempranas para las familias con niños con necesidades especiales. El desafío quedaría en diseñar un programa de intervención temprana que contenga los aspectos mencionados anteriormente y donde podamos cuantificar el impacto de éste tanto en el plano económico como en el niño y su familia.

\section{Agradecimientos a,}

Rodolfo Bastias, Kinesiólogo de la Unidad de Neonatología del Hospital Luis Tisne.

Marianela Hoffmann, Psicóloga Universidad del Desarrollo. 


\section{REFERENCIAS BIBLIOGRAFICAS.}

1 FORCADA-GUEX M, PIERREHUMBERT B, BORGHINI A, MOESSINGER A, MULLER-NIX C. Early dyadic patterns of mother-infant interactions and outcomes of prematurity at 18 months. Pediatrics 2006; 118: 107-114.

2 FIELDMAN R, EIDELMAN A. Neonatal state organization, neuromaturation, motherinfant interaction, and cognitive development in small-for-gestational-age premature infants. Pediatrics 2006; 118 (3): 869-878.

${ }^{3}$ YARNOZ S, PAEZ D. Una aproximación a los Modelos Interactivos Activos (IWM) de la Teoría del Apego como constructos sociocognitivos. Boletín de Psicología 1993; 41: 91-102

${ }^{4}$ LECANNELIER F. Apego, autorregulación y organización cerebral: las reflexiones integradoras de Allan Schore. En: LECANNELIER F, ed. Apego e intersubjetividad. Influencia de los vínculos tempranos en el desarrollo humano y la salud mental. Santiago de Chile: Editorial LOM Ediciones, 2006; 27-42.

${ }^{5}$ SCHORE A. The effects of a secure attachment relationship on right brain development, affect, regulation and infant mental health. Infant Mental Health Journal 2001; 22: 7-66.

${ }^{6}$ STERN M, KARRAKER K, MCINTOSH B, MORITZEN S, OLEXA M. Prematurity stereotyping and mothers'interaction with their premature and full term infant during the first year. Journal of Pediatric Psychology 2006; 31(6): 597-607.

${ }^{7}$ GREENSPAN S, WIEDER S. Discovering each child's unique strengths, developmental capacities and challenges. En: GREENSPAN S, WIEDER S, eds. The child with special needs. Encouraging intellectual and emotional growth. United States: Da Capo Press, 1998; 17-118.

8 WENDLAND-CARRO J, PICCININI C, MILLAR W. The role of an early intervention on enhancing the quality of mother-infant interaction. Child Development 1999; 70(3): 713-721.

${ }^{9}$ LEAVITT L. Mothers’ sensitivity to infant signals. Pediatrics1998; 102 (5): 1247-1249

${ }^{10}$ VERDEN-ZÖLLER G. El juego en la relación materno-infantil. Fundamento biológico de la conciencia de sí mismo y de la conciencia social. En: MATURANA H, VERDEN-ZÖLLER G. Eds. Amor y Juego: Fundamentos olvidados de lo humano. Santiago de Chile: Editorial Comunicaciones Noreste Ltda., 2007; 115-207.

${ }^{11}$ SCHAAF R, ANZALONE M. (2001). Sensory integration with high-risk infants and young children. En SMITH S, IMPERATORE E, SCHAAF R, eds. Understanding the nature of sensory integration with diverse populations. United States: Editorial Therapy Skill Builders, 2001; 257-312. 